\title{
Exponentially correlated Gaussian functions in variational calculations. Momentum space properties of the ground state helium dimer
}

\author{
Jacek Komasa ${ }^{\text {) }}$ \\ Quantum Chemistry Group, Faculty of Chemistry, A. Mickiewicz University, Grunwaldzka 6, \\ 60-780 Poznań, Poland
}

(Received 16 November 2000; accepted 30 March 2001)

\begin{abstract}
Microhartree accuracy wave functions composed of exponentially correlated Gaussians were transformed in closed form to momentum space representation and applied to compute various isotropic momentum space properties of helium dimer in the ground state. The set of properties includes electron momentum density distribution, expectation values of powers of the electronic momentum operator, and the Compton profile. Calculations were performed at many internuclear separations $R$ including the united atom (beryllium) and the separated atoms (helium) limits.

(C) 2001 American Institute of Physics. [DOI: 10.1063/1.1373428]
\end{abstract}

\section{INTRODUCTION}

The existence of stable $\mathrm{He}_{2}$ molecule was confirmed experimentally ${ }^{1-4}$ which immediately implies many questions concerning the electronic structure and properties of this system. A large number of ab initio calculations on the ground state of helium dimer that appeared only in the last two years demonstrates that it is a very intensively explored field of research. ${ }^{5-16}$ Among new theoretical papers there are several dealing with micro- and macroscopic properties of $\mathrm{He}_{2}$ often aiming at direct confrontation with the experimental data. The papers concern electric, ${ }^{17-20}$ magnetic, ${ }^{21}$ thermophysical, ${ }^{22}$ wave-optical, ${ }^{23}$ and low-temperature flow properties. $^{24}$

Full description of the electronic structure of molecules must include both position and momentum space properties. However, because of a relative scarcity of the momentum space data, they have brought much smaller contribution to the knowledge of the electronic structure of molecules than the position space properties. It is, therefore, desirable to develop this branch of the description of the molecular systems and phenomena. In particular, it is important to supply accurate data for simple systems which might serve as a reference for future work. As the hydrogen molecule in the past, the helium dimer today became a test case for development of new computational methods and tools. As a small but, for his exceptionally weak binding, particularly demanding system, the helium dimer is a real challenge for the most sophisticated quantum chemical methods aiming at high accuracy.

Since the wave functions of microhartree accuracy became available for helium dimer ${ }^{25,26}$ a possibility of computing reliable properties of this system has increased. In two preceding papers ${ }^{27,28}$ of the series devoted to properties of $\mathrm{He}_{2}$, the accurate expectation values of energy components and quadrupole moment operators were presented. The information complementary to the position space properties can be obtained in the momentum space and this opportunity is

a)Electronic mail: komasa@man.poznan.pl exploited in this work. Of particular interest is the manifestation of significant changes in the electronic structure of $\mathrm{He}_{2}$ at short internuclear distances, described in terms of the position space properties reported previously, ${ }^{27,28}$ in the momentum space.

Despite difficulties in determination of the momentum space wave function the field of the momentum space properties has enjoyed much interest. ${ }^{29-34}$ There are many reasons which make the momentum space properties so attractive. One surely comes from the fact that many of them is accessible from measurements. This opens a possibility of mutual verification of the experimental data and the model used in their interpretation on one side and the theory employed in the computations on the other; e.g., validity of the impulse approximation ${ }^{35}$ can be verified by a comparison of Compton profile measured in scattering process with that obtained theoretically from highly accurate calculations. ${ }^{36-38}$ The $x$-ray and $\gamma$-ray Compton scattering and electron scattering experiments allow also the radial moments $\left\langle p_{i}^{k}\right\rangle$ of momentum density distribution to be indirectly calculated and confronted with those known from theory.

The most common technique used to compute momentum space properties is based on Fourier transformation of the wave function obtained from the solution of the position space Schrödinger equation. For systems with a few electrons the highest quality solutions can be obtained using explicitly correlated wave functions, i.e., functions of the interelectron distance, $r_{i j}$. In the past, momentum space properties obtained from this type of wave functions have proven to be an important tool in interpreting physical phenomena. ${ }^{37,39}$ However, a wide class of the explicitly correlated molecular wave functions, namely those including odd powers of the interelectronic distance, cannot be transformed analytically to the momentum space representation. On the other hand, the linear $r_{i j}$ factor increases the rate of the energy convergence and is necessary to fulfill the cusp condition. The exponentially correlated Gaussian (ECG) wave functions allow this bottleneck to be circumvented. These wave functions with Gaussian correlation satisfy nei- 
ther the electron-nucleus nor the electron-electron cusp condition, however, they are capable of yielding very accurate energy and, simultaneously, undergo easily mathematical manipulations including analytic Fourier transformation. ${ }^{37}$

To the best of the author's knowledge there are no momentum space calculations concerning helium dimer reported in the literature and this gap should be filled up. The aim of this paper is to present analytic expressions for the correlated $n$-electron wave function in the momentum space (Sec. II B) and benchmark results for a variety of isotropic momentum space properties of $\mathrm{He}_{2}$ (Sec. III). Hopefully, the accurate theoretical data presented in this paper will be stimulating to the experimentalists, too.

\section{METHOD OF COMPUTATION}

\section{A. Position space wave function}

The ansatz for the $n$-electron $K$-term wave function reads

$$
\Psi(\mathbf{r}, \boldsymbol{\sigma})=\hat{\mathcal{A}}\left(\Xi_{n, S, M_{S}}(\boldsymbol{\sigma}) \hat{\mathcal{S}} \sum_{k=1}^{K} c_{k} \psi_{k}(\mathbf{r})\right),
$$

where $\hat{\mathcal{A}}$ and $\hat{\mathcal{S}}$ are permutation and spatial symmetry operators, respectively, and $\Xi_{n, S, M_{S}}$ is an $n$-electron spin function with the spin quantum numbers $S$ and $M_{S}\left(S=0\right.$ and $M_{S}$ $=0$ in the present case). $\boldsymbol{\sigma}$ and $\mathbf{r}$ are vectors of, respectively, spin and space coordinates of the electrons. Exponentially correlated Gaussian (ECG) functions of Singer ${ }^{40}$ were used as the $n$-electron spatial basis functions $\psi_{k}$ :

$$
\psi_{k}(\mathbf{r})=\exp \left[-\left(\mathbf{r}-\mathbf{s}_{k}\right) \mathbf{A}_{k}\left(\mathbf{r}-\mathbf{s}_{k}\right)^{T}\right],
$$

where the superscript $T$ denotes a vector transpose. The linear, $c_{k}$, and the nonlinear parameters, $A_{k, i j}$ and $s_{k, i}$, were determined in a variational optimization process. The nonlinear parameters were optimized in small $n(n+1) / 2$ dimensional subspaces. Each subspace was defined by parameters belonging to a single basis function. While the other parameters were kept fixed, the energy minimum in the subspace was located by means of Powell's method. ${ }^{41}$ Such a procedure was applied subsequently to all expansion terms, comprising a single cycle of the optimization. The energy gain from the cycle was taken as a measure of the convergence. For each internuclear distance, $R$, as many cycles was performed as needed to converge the energy gain to a fraction of microhartree.

Detailed description of the method as well as many expectation values (including energies) obtained with the wave functions applied in this work may be found in Refs. 25-28. We only note here that the error in the total BornOppenheimer energy curve obtained from the ECG wave functions applied in this work ranges from a fraction to a few tens of microhartree, ${ }^{27}$ depending on $R$, and this is the most accurate variational curve of $\mathrm{He}_{2}$ currently available.

The spatial part of the ECG wave function can be equivalently represented in the form introduced by Boys: ${ }^{42}$

$\psi_{k}(\mathbf{r})=\exp \left[-\sum_{i=1}^{n} a_{k, i}\left|\mathbf{r}_{i}-\mathbf{C}_{k, i}\right|^{2}-\sum_{i \neq j=1}^{n} b_{k, i j}\left|\mathbf{r}_{i}-\mathbf{r}_{j}\right|^{2}\right]$
Both forms of the ECG wave function are easily convertible to each other with the following relation of their nonlinear parameters:

$$
\begin{aligned}
b_{k, i j} & =-A_{k, i j} \quad(i \neq j), \\
a_{k, i} & =\sum_{j=1}^{n} A_{k, i j}, \\
\mathbf{C}_{k, i} & =\left(\sum_{j=1}^{n} A_{k, i j} \mathbf{s}_{i}\right) / a_{k, i} .
\end{aligned}
$$

In the past, the Boys form, having more transparent physical interpretation, was used rather than the form of Singer which in turn is more convenient from the mathematical point of view and which was subjected to the Fourier transformation presented below.

\section{B. Transformation to momentum space representation}

The wave function in the position space, $\Psi$, can be transformed to the momentum space representation by means of the Fourier integral

$$
\widetilde{\Psi}(\mathbf{p}, \boldsymbol{\sigma})=(2 \pi)^{-(3 / 2) n} \int \Psi(\mathbf{r}, \boldsymbol{\sigma}) \exp \left[-i \mathbf{p} \mathbf{r}^{T}\right] d \mathbf{r} .
$$

It has been known for a long time that for the two-electron atomic $^{43,44}$ and molecular ${ }^{37,45}$ ECG wave functions the integration in Eq. (5) can be carried out analytically. As will now be shown this is also true in the general case of the $n$-electron ECG wave function. Inserting Eqs. (1) and (2) into Eq. (5) we arrive at $\widetilde{\Psi}$ expanded in the basis of $n$-electron $\widetilde{\psi}_{k}$ functions. Such a Fourier transformed basis function $\widetilde{\psi}_{k}$ can be written down in closed form as:

$\widetilde{\psi}_{k}(\mathbf{p})=2^{-(3 / 2) n}\left|\mathbf{A}_{k}\right|^{-(3 / 2)} \exp \left[-\frac{1}{4} \mathbf{p} \mathbf{A}_{k}^{-1} \mathbf{p}^{T}-i \mathbf{s}_{k} \mathbf{p}^{T}\right]$

or, in analogy with Eq. (2), as:

$\widetilde{\psi}_{k}(\mathbf{p})=2^{3(1-n / 2)}\left|\widetilde{\mathbf{A}}_{k}\right|^{-(3 / 2)} \exp \left[-\left(\mathbf{p}-\widetilde{\mathbf{s}}_{k}\right) \widetilde{\mathbf{A}}_{k}\left(\mathbf{p}-\widetilde{\mathbf{s}}_{k}\right)^{T}+\widetilde{C}\right]$

with $\widetilde{\mathbf{s}}_{k}=-2 i \mathbf{s}_{k} \mathbf{A}_{k}, \widetilde{\mathbf{A}}_{k}=\frac{1}{4} \mathbf{A}_{k}^{-1}$, and $\widetilde{C}=-\mathbf{s}_{k} \mathbf{A}_{k} \mathbf{s}_{k}^{T}$. Using relations similar to those of Eq. (4), $\widetilde{\psi}_{k}$ can be expressed also in the Boys-like form.

The Fourier transformation preserves the shape and all the advantages of the original ECG function, which enables the evaluation of integrals in the momentum space to be readily accomplished. For instance, the expectation values of simple electronic momentum operators $p^{k}$ can be computed as easily as the expectation values of the position operators $r^{k}$ with $\Psi$.

\section{RESULTS AND DISCUSSION}

For an arbitrary one-electron operator $\hat{o}_{i}$ we use the shorthand notation

$$
\left\langle\hat{o}_{i}\right\rangle=\int \widetilde{\Psi}^{*}(\mathbf{p}, \boldsymbol{\sigma})\left(\sum_{i=1}^{n} \hat{o}_{i}\right) \widetilde{\Psi}(\mathbf{p}, \boldsymbol{\sigma}) d \mathbf{p} d \boldsymbol{\sigma}
$$

with the normalization to $n$-the number of electrons. As we work in the framework of the Born-Oppenheimer approxi- 


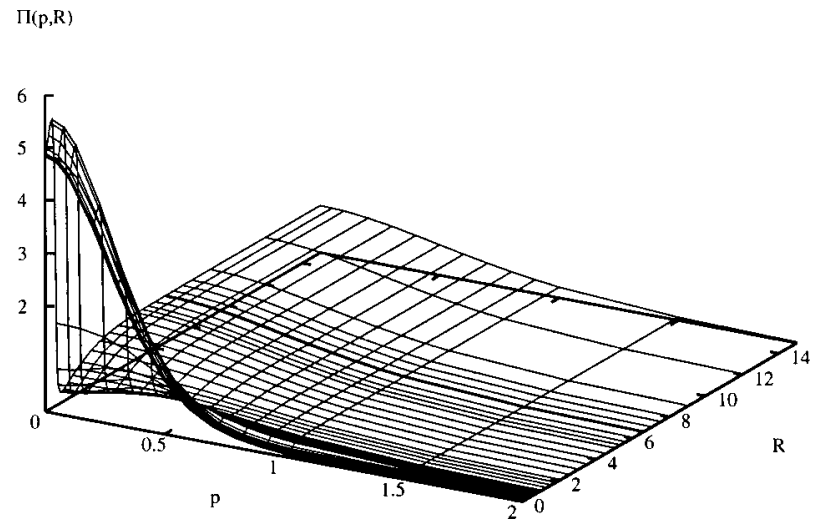

FIG. 1. Spherically averaged momentum density $\Pi(p, R)$ obtained from 1200-term ECG wave functions.

mation both the position and the momentum space wave functions depend parametrically on the internuclear distance, $R$. Hence, all the properties considered in this paper implicitly depend on $R$ as well.

Except for the separated atom limit, where the ECG wave function with $K=600$ was used, ${ }^{46}$ the 1200 -term expansion ${ }^{27}$ was applied for the whole range of $R$.

Throughout the atomic units are used.

\section{A. Electron momentum density distribution}

The distribution of the electronic momentum is often analyzed in terms of one-dimensional functions: the spherically averaged momentum density

$$
\Pi(p)=\frac{1}{4 \pi p^{2}}\left\langle\delta\left(p-p_{i}\right)\right\rangle
$$

or the radial momentum density

$$
I(p)=4 \pi p^{2} \Pi(p) .
$$

With the ECG wave function the integration in Eq. (9) can be performed in closed form leading to analytic expressions for $\Pi(p)$ and $I(p)$. For instance, the matrix elements of $\Pi(p)$ have the following simple functional dependence on $p$ :

$$
e^{-A p^{2}} \sin (B p) p^{-1} \text {. }
$$

The shape of the $\Pi(p ; R)$ surface is presented graphically in Fig. 1. A table with the numerical values was deposited in E-PAPS. ${ }^{47}$ The density surface smoothly connects the united atom and the separated atoms limits. It has a global maximum at $p=0.0, R=0.4$ and a saddle point around $p$ $=0.4, R=1.25$.

When the two nuclei approach each other, $\Pi(0)$ slowly decreases in order to reach a 0.68 deep minimum at $R=0.9$ and than to jump up to a maximum at $R=0.4$ (Fig. 2). This behavior can be explained on the basis of a joint momentum and position space picture. If we recall that the value of $\Pi(0)$ emphasizes the large $\mathbf{r}$ region, so that it can be employed as a measure of the size of the outermost $s$ orbital in atoms, ${ }^{48-50}$ we shall see that the above description indicates that in the range of $R<0.7$ the electrons are pulled off to the outer region of electron position density. This interpretation

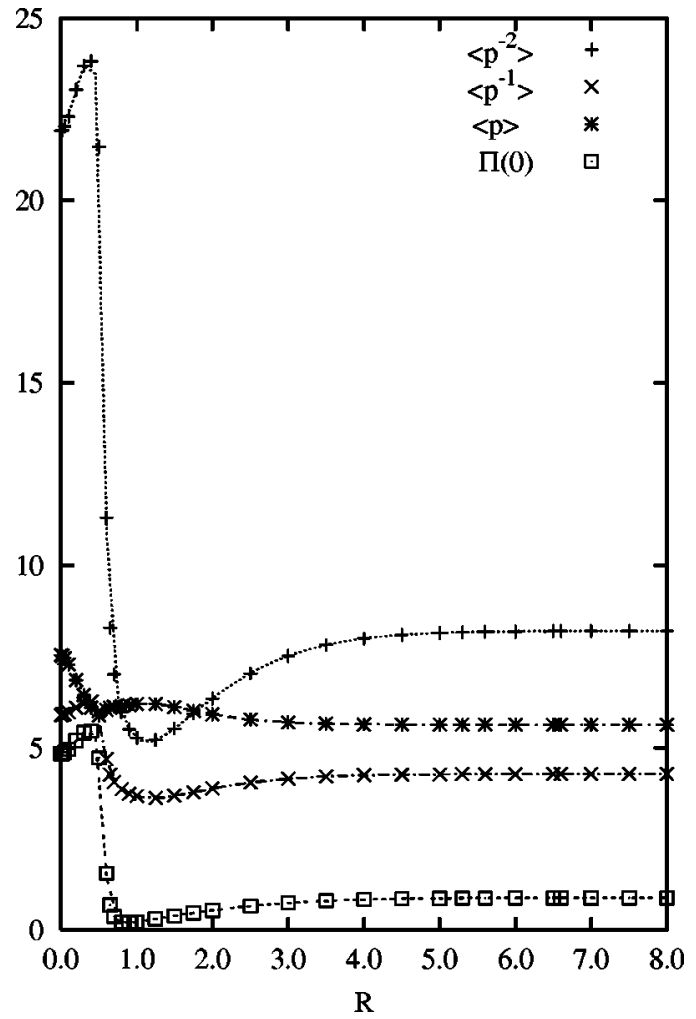

FIG. 2. $R$-dependence of $\left\langle p_{i}^{-2}\right\rangle,\left\langle p_{i}^{-1}\right\rangle,\left\langle p_{i}\right\rangle$, and $\Pi(0)$ obtained from 1200-term ECG wave functions.

is also supported by an observation that the shape of $\Pi(0)$ at small $R$ is consistent with behavior of the quadrupole moment expectation values reported in Ref. 28.

The exact spherically averaged momentum density is known $^{49,51,52}$ to behave as

$$
\Pi(p)=\Pi(0)+a_{2} p^{2}+a_{4} p^{4}+\mathcal{O}\left(p^{6}\right)
$$

for small $p$, and as

$$
\Pi(p)=A_{8} p^{-8}+A_{10} p^{-10}+A_{12} p^{-12}+\mathcal{O}\left(p^{-14}\right)
$$

for large values of momentum. The power series expansion coefficients at selected internuclear distances are listed in Table I. The parameters of the small- $p$ expansion Eq. (12) were determined from the fit of the parabola $a_{2}+a_{4} p^{2}$ to the points of the function $[\Pi(p)-\Pi(0)] / p^{2}$ (Ref. 52) computed in the range $(0,0.1\rangle$ with the step 0.01 . The parameters of the large momentum expansion Eq. (13) come from the fit to $\Pi\left(p_{j}\right)$ with $p_{j}=10,15,20,50,100$, and 200. The number of digits of the entries in Table I depends on the quality of the fit measured by means of the standard deviation of a particular parameter.

The $a_{2}=\Pi^{\prime \prime}(0) / 2$ parameter is positive in the range $0.626 \leq R /$ bohr $\leqslant 1.712$. The sign of the second derivative determines whether the critical point $(p=0)$ is a local minimum or maximum. For these values of $R$ where $a_{2}>0$, there is a local minimum at $p=0$ and $\Pi(p)$ must be nonmonotonic, i.e., exhibits a local maximum at some $p>0$. Indeed, the maxima appear around $p=0.4$. Westgate et al. ${ }^{48}$ in their study of momentum density of atoms and ions, called the density maxima appearing below $p=0.6$ and larger than the value of $\Pi(0)$ "slow.' They related the slow maxima to the 
TABLE I. Parameters of the small- and large- $p$ expansions [Eqs. (12) and (13)] of the spherically averaged momentum density. $X[Y]$ stands for $X \times 10^{Y}$.

\begin{tabular}{ccclccc}
\hline \hline$R /$ bohr & $\Pi(0)$ & \multicolumn{1}{c}{$a_{2}$} & $a_{4}$ & \multicolumn{1}{c}{$A_{8}$} & \multicolumn{1}{c}{$A_{10}$} & $A_{12}$ \\
\hline 0.00 & 4.834493 & $-3.4885[1]$ & $1.434[2]$ & $1.418[3]$ & $-9.36[5]$ & $2.59[6]$ \\
0.01 & 4.836795 & $-3.4922[1]$ & $1.437[2]$ & $1.408[3]$ & $-9.22[5]$ & $2.52[6]$ \\
0.10 & 4.955520 & $-3.6188[1]$ & $1.493[2]$ & $8.1[2]$ & $-8 .[4]$ & $-1.1[6]$ \\
0.40 & 5.456556 & $-4.2906[1]$ & $1.896[2]$ & $2.23[2]$ & $-2.68[5]$ & $1.73[6]$ \\
0.60 & 1.557194 & $-1.055[1]$ & $-3.56[1]$ & $8.6[1]$ & $7.0[3]$ & $7.69[6]$ \\
0.80 & 0.217022 & 7.133 & $-5.21[1]$ & $7.4[1]$ & $5 .[3]$ & $-3 .[5]$ \\
1.00 & 0.225636 & 3.2119 & $-1.858[1]$ & $9.9[1]$ & $-6.8[3]$ & $3.64[6]$ \\
1.25 & 0.304694 & 1.13244 & -5.70 & $7.26[1]$ & $8 .[2]$ & $-1.48[6]$ \\
1.50 & 0.388865 & 0.35514 & -2.410 & $8.10[1]$ & $-2.03[3]$ & $5.1[5]$ \\
2.00 & 0.540730 & -0.337408 & -0.564 & $7.6295[1]$ & $-1.099[3]$ & $1.90[5]$ \\
3.00 & 0.744174 & -1.03724 & 0.7204 & $7.382[1]$ & $-1.18[3]$ & $-2 .[5]$ \\
4.00 & 0.836110 & -1.38513 & 1.354 & $7.400[1]$ & $-1.340[3]$ & $7.0[3]$ \\
5.00 & 0.870076 & -1.54375 & 1.681 & $7.4090[1]$ & $-1.3985[3]$ & $1.165[4]$ \\
5.60 & 0.878241 & -1.59083 & 1.793 & $7.4051[1]$ & $-1.3897[3]$ & $1.112[4]$ \\
6.00 & 0.881245 & -1.61050 & 1.843 & $7.4085[1]$ & $-1.4005[3]$ & $1.184[4]$ \\
7.00 & 0.884709 & -1.63735 & 1.923 & $7.4106[1]$ & $-1.405[3]$ & $1.211[4]$ \\
8.00 & 0.885764 & -1.64790 & 1.960 & $7.4121[1]$ & $-1.410[3]$ & $1.241[4]$ \\
12.00 & 0.886254 & -1.65541 & 2.002 & $7.4128[1]$ & $-1.411[3]$ & $1.253[4]$ \\
15.00 & 0.886221 & -1.65492 & 1.999 & $7.4130[1]$ & $-1.412[3]$ & $1.259[4]$ \\
$\infty$ & 0.886256 & -1.65503 & 1.994 & $7.4108[1]$ & $-1.4060[3]$ & $1.218[4]$ \\
\hline \hline
\end{tabular}

outermost $p$-shell electrons. In this work a similar shellcontribution analysis cannot be performed because the ECG ansatz is free of the one-electron approximation. Instead, let us notice that the $R$-interval of the slow maximum coincides with that of the smallest values of $\Pi(0)$ and can be ascribed the same physical origin, i.e., the contraction of the position density. This contraction of space accessible for the electrons increases the probability density of finding electrons with higher average momentum.

Recently, Koga and co-workers ${ }^{50}$ suggested the following classification of atomic $\Pi(p)$ functions: $\Pi(p)$ with a single maximum at $p=0$ (Type I), $\Pi(p)$ with a single maximum at $p>0$ (Type II), and $\Pi(p)$ with both maxima (Type III). Assuming that this classification can be extended to molecular densities, the momentum density inside the critical $R$-interval belongs to Type II and outside to Type I.

\section{B. Moments of the electron momentum density}

The asymptotic behavior of the exact $\Pi(p)$ and its finite nonzero value at $p=0$ implies a limitation on $k$ in $\left\langle p_{i}^{k}\right\rangle$ and it can be shown that this expectation value is well defined only for $-2 \leqslant k \leqslant 4$. The moments are particularly interesting for their relation to many other physical quantities: ${ }^{53-56}$ $\left\langle p_{i}^{-1}\right\rangle$ is twice the height of the Compton profile at the origin, $\left\langle p_{i}^{0}\right\rangle$ evaluates to the number of electrons, $\left\langle p_{i}\right\rangle$ is related to the average magnitude of the current density and the exchange energy of Density Functional Theory, $\left\langle p_{i}^{2}\right\rangle$ is just twice the kinetic energy of the system which in combination with the virial theorem may be employed as an independent source of the total energy of the system, $\left\langle p_{i}^{3}\right\rangle$ is often linked with the initial value of the Patterson function widely used in crystallography, and finally, $\left\langle p_{i}^{4}\right\rangle$ enters the Breit-Pauli relativistic correction to the kinetic energy originating from the variation of mass with velocity. Another example is the ex- pectation value of $\mathbf{p}_{i} \mathbf{p}_{j}$ which is involved in the $S(1)$ sum rule and when combined with $\left\langle p_{i}^{2}\right\rangle$ leads to two-electron operator expectation value $\left\langle p_{i j}^{2}\right\rangle$.

Analytically evaluated $\left\langle p_{i}^{k}\right\rangle$ with odd $k$ lead to the following integral:

$$
G_{J}(\alpha, \beta)=\int_{0}^{1} s^{2 J} e^{\alpha s^{2}-\beta} d s,
$$

which is a generalization of the Boys function, ${ }^{42,57,58} F_{J}(t)$ $=\int_{0}^{1} s^{2 J} e^{-t s^{2}} d s, t \geqslant 0$, well known in the standard quantum chemical calculation, and of the Dawson's integral $\int_{0}^{1} s^{2 J} e^{t\left(s^{2}-1\right)} d s$ appearing in inverse-square distance potential integrals. ${ }^{59}$ To ensure accurate and rapid evaluation of $G_{J}(\alpha, \beta)$ the algorithm of McMurchie and Davidson ${ }^{57,59}$ was adopted to positive values of $\alpha$.

The expectation values of the electron momentum density moments at selected internuclear distances are listed in Table II and drawn in Figs. 2 and 3. The $\left\langle p_{i}^{-2}\right\rangle$ and $\left\langle p_{i}^{-1}\right\rangle$ curves differ in shape from the other radial momentum curves. They exhibit global maxima and minima at small $R$ : around $R=0.4$ bohr and $R=1.2 \mathrm{bohr}$, respectively, and in this way they mimic the $\Pi(0)$ curve of Fig. 2 . The momentum operators with negative powers sample the momentum space density distribution near the origin and the outer region of the position space density. Analogously to $\Pi(0)$ curve they can be employed to measure effectively the electron cloud size and to observe the variations in the r-space density distribution with changing internuclear distance.

The remaining $\left\langle p_{i}^{k}\right\rangle$ curves reach global maxima at the united atom limit. They are monotonic except the $\left\langle p_{i}\right\rangle$ curve which has a local minimum at $R=0.5 \mathrm{bohr}$ and a local maximum around $R=1.2 \mathrm{bohr}$. At small $R$, this curve follows the shape of the electron-electron repulsion energy and the parallel component of the kinetic energy curves. ${ }^{27}$ The $\left\langle p_{i}^{2}\right\rangle$ 
TABLE II. Moments of the electron momentum density distribution calculated from 1200-term ECG wave function of $\mathrm{He}_{2}$.

\begin{tabular}{rrcccccr}
\hline \hline$R /$ bohr & $\left\langle p_{i}^{-2}\right\rangle$ & $\left\langle p_{i}^{-1}\right\rangle$ & $\left\langle p_{i}\right\rangle$ & $\left\langle\mathbf{p}_{i} \mathbf{p}_{j}\right\rangle$ & $\left\langle p_{i}^{2}\right\rangle$ & $\left\langle p_{i}^{3}\right\rangle$ & $\left\langle p_{i}^{4}\right\rangle$ \\
\hline 0.00 & 21.9068 & 5.90316 & 7.533780 & 0.4602293 & 29.33471 & 186.3700 & 2162.7 \\
0.01 & 21.9133 & 5.90418 & 7.530104 & 0.4599735 & 29.29259 & 185.5245 & 2086.9 \\
0.05 & 22.0309 & 5.92403 & 7.456369 & 0.4547528 & 28.48903 & 173.7124 & 1753.1 \\
0.10 & 22.2973 & 5.97107 & 7.283305 & 0.4418836 & 26.73978 & 152.9070 & 1389.9 \\
0.40 & 23.8178 & 6.26829 & 6.090424 & 0.3042701 & 17.01387 & 70.7777 & 474.2 \\
0.60 & 11.3033 & 4.68624 & 6.031135 & -0.2619697 & 14.32428 & 50.7843 & 306.9 \\
0.80 & 5.9724 & 3.86576 & 6.144458 & -0.5722651 & 13.60893 & 44.4338 & 255.6 \\
1.00 & 5.2597 & 3.67030 & 6.194003 & -0.5476394 & 13.42869 & 42.7013 & 242.5 \\
1.25 & 5.2106 & 3.62172 & 6.192009 & -0.3481395 & 13.28611 & 41.8921 & 238.6 \\
1.50 & 5.5276 & 3.68325 & 6.116329 & -0.1345578 & 13.00549 & 40.9271 & 234.7 \\
2.00 & 6.3513 & 3.87825 & 5.917948 & 0.1340853 & 12.37802 & 38.9621 & 226.0 \\
3.00 & 7.5138 & 4.14774 & 5.695327 & 0.2918272 & 11.76058 & 37.1836 & 217.5 \\
4.00 & 7.9895 & 4.24398 & 5.640199 & 0.3147138 & 11.63415 & 36.8606 & 216.2 \\
5.00 & 8.1416 & 4.26982 & 5.630570 & 0.3177102 & 11.61644 & 36.8211 & 216.2 \\
5.60 & 8.1734 & 4.27439 & 5.629495 & 0.3180115 & 11.61498 & 36.8185 & 216.2 \\
6.00 & 8.1841 & 4.27578 & 5.629264 & 0.3180788 & 11.61476 & 36.8183 & 216.2 \\
6.50 & 8.1916 & 4.27666 & 5.629165 & 0.3181138 & 11.61472 & 36.8184 & 216.2 \\
7.00 & 8.1953 & 4.27706 & 5.629143 & 0.3181267 & 11.61476 & 36.8186 & 216.2 \\
8.00 & 8.1982 & 4.27732 & 5.629149 & 0.3181346 & 11.61483 & 36.8188 & 216.2 \\
12.00 & 8.1992 & 4.27738 & 5.629168 & 0.3181385 & 11.61489 & 36.8190 & 216.2 \\
15.00 & 8.1991 & 4.27737 & 5.629170 & 0.3181384 & 11.61490 & 36.8190 & 216.2 \\
$\infty$ & 8.1992 & 4.27738 & 5.629170 & 0.3181390 & 11.61490 & 36.8191 & 216.3 \\
\hline \hline
\end{tabular}

curve and their Cartesian components were already discussed in Ref. 27.

The knowledge of $\left\langle p_{i}^{4}\right\rangle$, in connection with electronnucleus $\left\langle\delta_{i a}\right\rangle$ and electron-electron $\left\langle\delta_{i j}\right\rangle$ Dirac-delta expectation values, enables in principle an evaluation of the relativistic correction of $\mathcal{O}\left(c^{-2}\right)$ ( $c$ is speed of light) in Dirac-

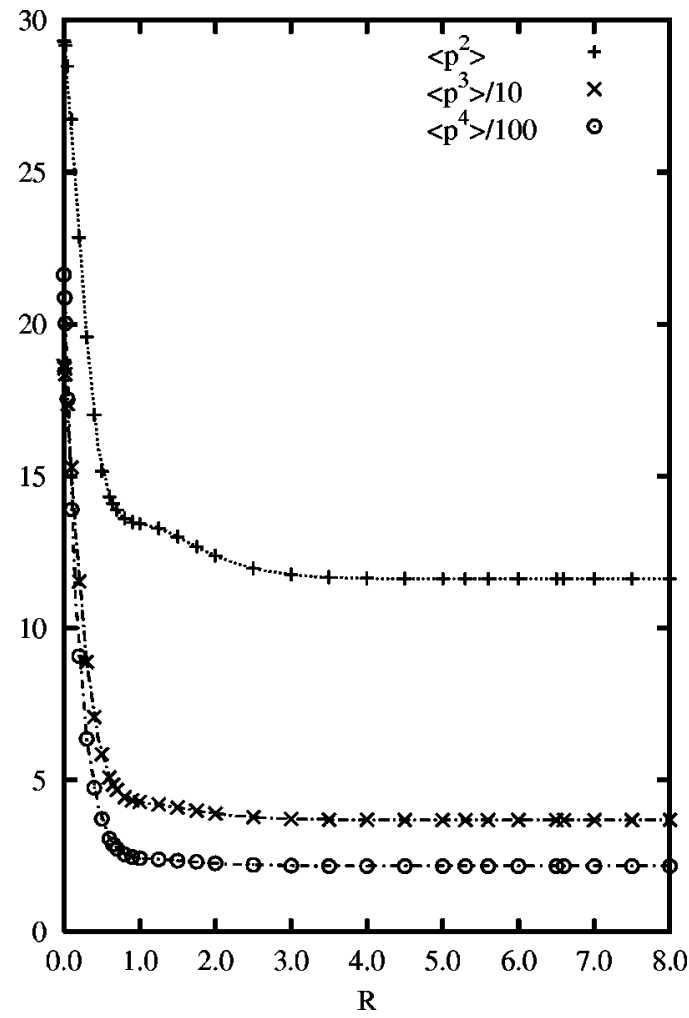

FIG. 3. $R$-dependence of $\left\langle p_{i}^{2}\right\rangle,\left\langle p_{i}^{3}\right\rangle$, and $\left\langle p_{i}^{4}\right\rangle$ obtained from 1200-term ECG wave functions.
Coulomb approximation, i.e., without the Breit correction (see, e.g., Ref. 46). Such preliminary calculations yield the relativistic correction to the total energy of about -0.26 millihartree at all $R \geqslant 4.0 \mathrm{bohr}$. It is more interesting, however, what is the influence of the relativistic effects on the $\mathrm{He}-\mathrm{He}$ interaction energy curve and the binding energy of this extremely weakly bound system. Unfortunately, in the present attempt, the accuracy of the Dirac-delta expectation values calculated with 1200-term ECG turned out to be too low to give a quantitative answer and still more accurate wave functions have to be employed to solve this problem.

In order to assess the accuracy of the computed moments an analysis of the convergence versus the length, $K$, of the wave function expansion was performed. The convergence was checked at $R=0.0, R=0.6, R=5.6^{60}$ and $R=\infty$. The most accurate are the expectation values of $p_{i}, \mathbf{p}_{i} \mathbf{p}_{j}, p_{i}^{2}$, and $p_{i}^{3}$ operators as 6-7 significant figures can be recognized stable. To 5 significant figures converge the $\left\langle p_{i}^{-1}\right\rangle$ and $\left\langle p_{i}^{-2}\right\rangle$. The worse accuracy is observed for $\left\langle p_{i}^{4}\right\rangle$, but still 4 digits remain stable.

As there is lack of published data available, the moments of the electron momentum density of $\mathrm{He}_{2}$ could not be confronted with the corresponding literature values. Only the limit cases of $R=0$ (beryllium atom) and $R=\infty$ (helium atoms) give an opportunity to compare the results of this work with literature data. In 1992 Tripathi et al. ${ }^{61}$ presented configuration-interaction results of the momentum space properties of beryllium atom. A few years later Meyer et $a l^{62}$ computed $\left\langle p_{i}^{k}\right\rangle(-2 \leqslant k \leqslant 2)$ from MR-SDCI wave function of Be. There are also numerical Hartree-Fock results available for both $\mathrm{Be}$ and $\mathrm{He} .{ }^{63}$ Correlated expectation values for helium atom were computed by Regier and Thakkar with 40-term Gaussian geminals. ${ }^{44}$ Also experimental data are available from the work of Lee. ${ }^{64}$ The abovemen- 
TABLE III. Comparison of $\left\langle p_{i}^{k}\right\rangle$ values obtained from the ECG wave functions for He and Be with literature data.

\begin{tabular}{|c|c|c|c|c|c|c|}
\hline Method & $\left\langle p_{i}^{-2}\right\rangle$ & $\left\langle p_{i}^{-1}\right\rangle$ & $\left\langle p_{i}\right\rangle$ & $\left\langle p_{i}^{2}\right\rangle$ & $\left\langle p_{i}^{3}\right\rangle$ & $\left\langle p_{i}^{4}\right\rangle$ \\
\hline \multicolumn{7}{|c|}{$\mathrm{He}$} \\
\hline 600-term ECG ${ }^{\mathrm{a}}$ & 4.099607 & 2.138688 & 2.814585 & 5.807449 & 18.40954 & 108.1698 \\
\hline 40-term $\mathrm{GG}^{\mathrm{b}}$ & 4.0986 & 2.13860 & 2.81459 & 5.80740 & 18.4056 & 106.56 \\
\hline 616-term $\mathrm{VEC}^{\mathrm{c}}$ & - & - & - & 5.807449 & - & 108.17613 \\
\hline 204-term $\mathrm{VEC}^{\mathrm{d}}$ & 4.08169 & 2.13682 & 2.81463 & 5.80682 & 18.40283 & 108.04796 \\
\hline $\mathrm{CI}^{\mathrm{e}}$ & - & 2.13596 & 2.8147 & 5.8063 & - & 108.59 \\
\hline $\mathrm{NHF}^{\mathrm{f}}$ & 4.0923 & 2.1410 & 2.7990 & 5.7234 & 17.990 & 105.63 \\
\hline Experiment $^{\mathrm{g}}$ & $4.115 \pm 0.144$ & \multicolumn{2}{|r|}{$\mathrm{Be}$} & $5.818 \pm 0.291$ & $17.11 \pm 3.42$ & $67.05 \pm 67.0$ \\
\hline 1200 -term $\mathrm{ECG}^{\mathrm{h}}$ & 21.906848 & 5.903158 & 7.533780 & 29.33471 & 186.3672 & 2162.698 \\
\hline 650-term $\mathrm{CI}^{\mathrm{i}}$ & 21.9387 & 5.9076 & 7.5330 & 29.3329 & - & - \\
\hline MRSDCI $^{\mathrm{j}}$ & 21.9287 & 5.9050 & 7.5334 & 29.3312 & - & - \\
\hline $\mathrm{NHF}^{\mathrm{f}}$ & 25.294 & 6.3185 & 7.4342 & 29.146 & 185.55 & 2158.7 \\
\hline
\end{tabular}

${ }^{a}$ This work, the ECG wave function of Ref. 46, E(He) $=-2.903724377$ hartree.

${ }^{\mathrm{b}}$ Correlated calculations with 40-term Gaussian geminals by Regier and Thakkar (Ref. 44), E(He) $=-2.903701$ hartree.

${ }^{c}$ Variational explicitly correlated wave function of Drake (Ref. 74), $\mathrm{E}(\mathrm{He})=-2.903724377034073$ hartree.

${ }^{\mathrm{d}}$ Variational explicitly correlated wave function of Arias de Saavedra et al. (Ref. 75), E(He) $=-2.903520$ hartree.

e35-term configuration interaction calculations by Banyard and Moore (Ref. 67), $\mathrm{E}(\mathrm{He})=-2.90320$ hartree.

${ }^{\mathrm{f}}$ Numerical Hartree-Fock calculations by Koga and Thakkar (Ref. 63), $\mathrm{E}(\mathrm{He})=-2.861679996$ hartree, $\mathrm{E}(\mathrm{Be})=-14.57302317$ hartree.

gObtained by fitting the measured Compton profile (Ref. 64).

${ }^{\mathrm{h}}$ This work, the ECG wave function of Ref. 76, $\mathrm{E}(\mathrm{Be})=-14.667355022$ hartree.

${ }^{\mathrm{i}}$ Configuration interaction calculations by Tripathi et al. (Ref. 61), $\mathrm{E}(\mathrm{Be})=-14.666902$ hartree.

${ }^{\mathrm{j}}$ Multi-reference singly and doubly excited configuration interaction calculations by Meyer et al. (Ref. 62), $\mathrm{E}(\mathrm{Be})=-14.66629$ hartree.

tioned results are specified in Table III and compared with the present work. For both atoms also the $\left\langle\mathbf{p}_{i} \mathbf{p}_{j}\right\rangle$ values can be found in literature. For beryllium 0.460229 from the present work can be compared with 0.4617 inferred from Ref. 65. For helium 0.159069 of Pekeris, ${ }^{66} 0.15735$ of Banyard and Moore, ${ }^{67}$ and 0.15907 of Froelich and Alexander ${ }^{68}$ can be compared with 0.1590695 obtained from the 600term ECG wave function.

Gálvez and Porras ${ }^{69}$ obtained inequalities limiting from above the spherically averaged momentum density of an atom

$$
\Pi(p) \leqslant \frac{k\left\langle p_{i}^{k-3}\right\rangle}{4 \pi p^{k}} \quad(1 \leqslant k \leqslant 7) .
$$

These bounds were constructed under the assumption that $\Pi(p)$ is a monotonically decreasing function of $p$. Both atoms, Be and $\mathrm{He}$, fulfill this condition and inequality (15) can be verified using numerical data represented in this work. This inequality happens to be fulfilled also for all $\mathrm{He}-\mathrm{He}$ internuclear distances considered in the present work which suggests that Eq. (15) may hold also for molecular systems.

\section{Compton profile}

In the frames of the impulse approximation ${ }^{35}$ the isotropic Compton profile is defined as

$$
J(q)=2 \pi \int_{|q|}^{\infty} p \Pi(p) d p .
$$

The following well-known equations relate the isotropic Compton profile to the moments of the electron momentum density: ${ }^{70}$

$$
\begin{aligned}
& \left\langle p_{i}^{k}\right\rangle=2(k+1) \int_{0}^{\infty} q^{k} J(q) d q \quad(0 \leqslant k \leqslant 4), \\
& \left\langle p_{i}^{-1}\right\rangle=2 J(0), \\
& \left\langle p_{i}^{-2}\right\rangle=2 \int_{0}^{\infty} q^{-2}[J(0)-J(q)] d q,
\end{aligned}
$$

which gives an excellent opportunity to mutually verify the computed quantities. Equations (17)-(19) are also of particular interest because they allow the experimentally accessible Compton scattering data to be, at least formally, translated into quantities which characterize a distribution of electron momentum in a molecule.

The integral Eq. (16) was evaluated in three different ways and checked against each other. First, a direct numerical integration in two separate intervals $(0, c)$ and $(c, \infty)$ was performed. The integrand for the matrix element of Compton profile was

$$
e^{-A p^{2}} \sin (B p) \text {. }
$$

The splitting parameter $c=400$ was chosen to minimize collectively the differences between $\left\langle p_{i}^{k}\right\rangle$ obtained analytically and from the relation

$$
\left\langle p^{k}\right\rangle=4 \pi \int_{0}^{\infty} p^{k+2} \Pi(p) d p \quad(-2 \leqslant k \leqslant 4)
$$

evaluated numerically. In the finite $(0, c)$ interval the piecewise Gauss-Lagrange quadrature was applied, in $(c, \infty)$ - the 64-point Gauss-Laguerre quadrature.

In the second method the integration domain in Eq. (16) was converted from $(|q|, \infty)$ to $(0,1)$ interval by a complex variable substitution. ${ }^{71}$ The imaginary part was subsequently ruled out leading to the following integral: 


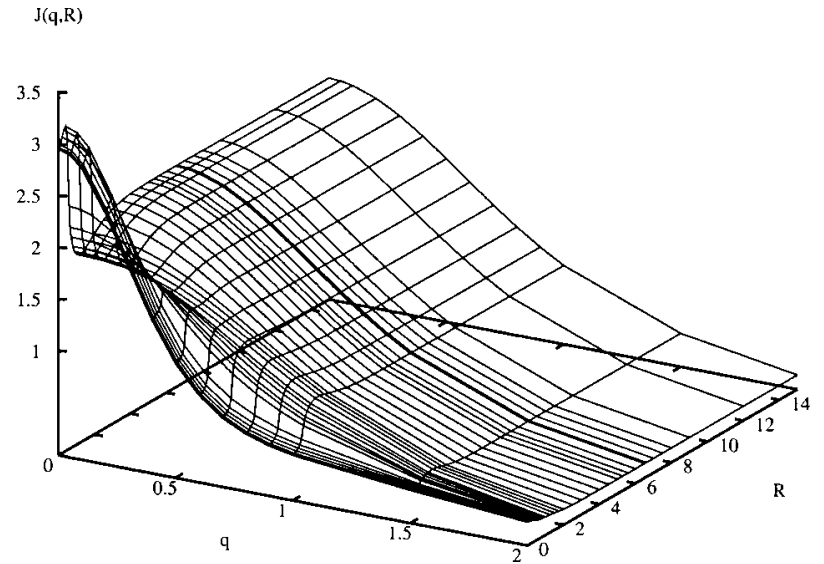

FIG. 4. Compton profile $J(q, R)$ obtained from 1200-term ECG wave functions.

$$
\int_{0}^{1} e^{-A q^{2} t^{2}+B t^{2}}\left[\cos \left(C q t^{2}\right)-D q^{2} t^{2} \sin \left(C q t^{2}\right)\right] d t
$$

evaluated by means of the 128-point Gauss quadrature.

Finally, in the third, analytic approach, the matrix elements of the Compton profile were expressed in terms of Faddeeva function ${ }^{72} w(z)=e^{-z^{2}} \operatorname{erfc}(-i z)$, where erfc is the complementary error function, as proposed in Ref. 71. The $w(z)$ function was evaluated according to the algorithm given by Poppe and Wijers. ${ }^{73}$

A relative difference between pairs of these three methods never exceeded $10^{-10}$ and, with respect to the accuracy, the methods described above can be recognized equivalent. However, evaluation times for these three methods with 1200-term wave function of $\mathrm{He}_{2}$ were approximately like 8:2:1 for a single $q$, and 6:12:1 for a simultaneous evaluation of $J(q)$ at a collection of 25 values of $q$.

The Compton profile is depicted in Fig. 4. Numerical values of $J(q ; R)$ are available from E-PAPS. ${ }^{47}$ The peak of the profile $J(0)$ as a function of $R$ has a global maximum at $R=0.4$ bohr and global minimum around $R=1.25$ bohr. Going from this minimum toward large separations the profile peak grows monotonically to the separated atoms limit. The largest variations in $J(q)$ appear at small $R$. For $q>0$, Eq. (27) below predicts that $d J / d q<0$. Indeed, in the $q$-direction the computed profile is monotonically decreasing for all $R$ and, contrary to the momentum density surface, has no saddle points.

In a similar way as $\Pi(p)$ in Eqs. (12) and (13), the isotropic Compton profile can be represented in a power series

$$
J(q)=\frac{1}{2}\left\langle p_{i}^{-1}\right\rangle+b_{2} q^{2}+b_{4} q^{4}+b_{6} q^{6}+\mathcal{O}\left(q^{8}\right)
$$

for small $q$, and as

$$
J(q)=B_{6} q^{-6}+B_{8} q^{-8}+B_{10} q^{-10}+\mathcal{O}\left(q^{-12}\right)
$$

for large $q$. The following simple equations, ${ }^{49}$

$$
b_{2 j}=-\frac{\pi}{j} a_{2 j-2} \quad(j \geqslant 1),
$$

$$
B_{2 j}=+\frac{\pi}{j} A_{2 j+2} \quad(j \geqslant 3),
$$

bind the expansion parameters of the Compton profile with those of the momentum density quoted in Table I.

Experimental Compton profile is most accurate near the peak $(q=0)$, therefore, modeling of this region is of particular interest. Note that

$$
\frac{d J}{d q}=-2 \pi q \Pi(q)
$$

and at $q=0$ the derivative vanishes. This suggests that around $q=0$ the Compton profile can be modeled by the Gaussian distribution

$$
J(q)=c_{1} e^{-c_{2} q^{2}} .
$$

From Eq. (18) we immediately have $c_{1}=\frac{1}{2}\left\langle p_{i}^{-1}\right\rangle$, while $c_{2}$ can be determined either from the fit to the small- $q$ values of $J(q)$ or using Eq. (27) and known value of $\Pi(q)$ at a small $q$.

\section{SUMMARY}

It was shown that the $n$-electron ECG wave function can be Fourier transformed in closed form to the momentum space representation retaining all the advantages of the Gaussian type functions. In the basis of the ECG functions the matrix elements of the momentum space properties (including the Compton profile) can be evaluated analytically using standard or in some cases special mathematical functions, hence, the time consuming numerical integration can be avoided. The wave functions used in this work yield energies accurate up to a fraction of microhartree. High quality of the wave functions allows the momentum space properties recommended in this work to be treated as benchmarks.

\section{ACKNOWLEDGMENTS}

I am indebted to Professor J. Rychlewski and Dr. W. Cencek for helpful comments and discussions. This study was supported by the Polish Research Committee Grants Nos. 3 T09A 08716 and SPUB-M/COST/D9, and by the computing grant from the Poznan Supercomputing and Networking Center.

${ }^{1}$ F. Luo, C. McBane, G. Kim, C. F. Giese, and W. R. Gentry, J. Chem. Phys. 98, 3564 (1993).

${ }^{2}$ W. Schöllkopf and J. P. Toennies, Science 266, 1345 (1994).

${ }^{3}$ W. Schöllkopf and J. P. Toennies, J. Chem. Phys. 104, 1155 (1996).

${ }^{4}$ F. Luo, C. F. Giese, and W. R. Gentry, J. Chem. Phys. 104, 1151 (1996).

${ }^{5}$ M. J. Jamieson, Chem. Phys. Lett. 310, 222 (1999).

${ }^{6}$ H. Partridge and C. W. Bauschlicher, Jr., Mol. Phys. 96, 705 (1999).

${ }^{7}$ S. M. Cybulski and R. R. Toczyłowski, J. Chem. Phys. 111, 10520 (1999).

${ }^{8}$ J. H. van Lenthe and F. Dijkstra, Mol. Phys. 96, 555 (1999).

${ }^{9}$ R. J. Gdanitz, Mol. Phys. 96, 1423 (1999).

${ }^{10}$ J. van de Bovenkamp and F. B. van Duijneveldt, J. Chem. Phys. 110, 11141 (1999).

${ }^{11}$ T. van Mourik and T. H. Dunning, Jr., J. Chem. Phys. 111, 9248 (1999).

${ }^{12}$ T. van Mourik, A. K. Wilson, and T. H. Dunning, Jr., Mol. Phys. 96, 529 (1999).

${ }^{13}$ J. Komasa, W. Cencek, and J. Rychlewski, Chem. Phys. Lett. 304, 293 (1999).

${ }^{14}$ J. M. Pérez-Jordá, E. San-Fabián, and A. Pérez-Jiménez, J. Chem. Phys. 110, 1916 (1999). 
${ }^{15}$ J. S. Lee and S. Y. Park, J. Chem. Phys. 112, 10746 (2000).

${ }^{16}$ R. J. Gdanitz, J. Chem. Phys. 113, 5145 (2000).

${ }^{17}$ L. W. Bruch, J. Chem. Phys. 112, 9773 (2000).

${ }^{18}$ C. Hättig, H. Larsen, J. Olsen, P. Jørgensen, H. Koch, B. Fernández, and A. Rizzo, J. Chem. Phys. 111, 10099 (1999).

${ }^{19}$ H. Koch, C. Hättig, H. Larsen, J. Olsen, P. Jørgensen, B. Fernández, and A. Rizzo, J. Chem. Phys. 111, 10108 (1999).

${ }^{20}$ M. Jaszuński, W. Klopper, and J. Noga, J. Chem. Phys. 113, 71 (2000)

${ }^{21}$ L. W. Bruch and F. Weinhold, J. Chem. Phys. 113, 8667 (2000).

${ }^{22}$ J. J. Hurly and M. R. Moldover, J. Phys. Chem. Ref. Data 28, 1713 (1999).

${ }^{23}$ G. C. Hegerfeldt and T. Köhler, Phys. Rev. A 61, 023606 (2000).

${ }^{24}$ L. Pademonte, G. Bracco, and R. Tatarek, Phys. Rev. A 59, 3084 (1999).

${ }^{25}$ J. Komasa and J. Rychlewski, Chem. Phys. Lett. 249, 253 (1996).

${ }^{26}$ J. Komasa and J. Rychlewski, Mol. Phys. 91, 909 (1997).

${ }^{27}$ J. Komasa, J. Chem. Phys. 110, 7909 (1999).

${ }^{28}$ J. Komasa, J. Chem. Phys. 112, 7075 (2000).

${ }^{29}$ A. Sarsa, F. J. Gálvez, and E. Buendía, J. Chem. Phys. 110, 5721 (1999).

${ }^{30}$ R. R. Zope, M. K. Harbola, and R. K. Pathak, Eur. Phys. J. D 7, 151 (1999).

${ }^{31}$ K. B. Joshi, R. Jain, R. K. Pandya, B. L. Ahuja, and B. K. Sharma, J. Chem. Phys. 111, 163 (1999).

${ }^{32}$ T. Koga, E. Romera, J. S. Dehesa, H. Matsuyama, and A. J. Thakkar, Theor. Chem. Acc. 103, 70 (1999).

${ }^{33}$ T. Koga and H. Matsuyama, Theor. Chem. Acc. 102, 39 (1999).

${ }^{34}$ T. Koga and H. Matsuyama, J. Chem. Phys. 111, 9191 (1999).

${ }^{35}$ B. G. Williams, Ed., Compton Scattering (McGraw-Hill, New York, 1977).

${ }^{36}$ V. H. Smith, Jr., A. J. Thakkar, W. H. Henneker, J. W. Liu, B. Liu, and R. E. Brown, J. Chem. Phys. 67, 3676 (1977).

${ }^{37}$ B. Jeziorski and K. Szalewicz, Phys. Rev. A 19, 2360 (1979).

${ }^{38}$ R. O. Esquivel, A. N. Tripathi, R. P. Sagar, and V. H. Smith, Jr., J. Phys. B 25, 2925 (1992).

${ }^{39}$ R. Benesch and V. H. Smith, Jr., Wave Mechanics: The First Fifty Years, edited by W. C. Price (Butterworths, London, 1973).

${ }^{40}$ K. Singer, Proc. R. Soc. London, Ser. A 258, 412 (1960).

${ }^{41}$ M. J. D. Powell, Comput. J. (UK) 7, 155 (1964).

${ }^{42}$ S. F. Boys, Proc. R. Soc. London, Ser. A 258, 402 (1960).

${ }^{43}$ P. E. Regier and A. J. Thakkar, Phys. Rev. A 30, 30 (1984).

${ }^{44}$ P. E. Regier and A. J. Thakkar, J. Phys. B 18, 3061 (1985).

${ }^{45}$ M. G. Henderson and C. W. Scherr, Phys. Rev. 120, 150 (1960).

${ }^{46}$ W. Cencek and W. Kutzelnigg, J. Chem. Phys. 105, 5878 (1996).

${ }^{47}$ See EPAPS Document No. E-JCPSA6-114-304124 for tables with $\Pi(p ; R)$ and $J(q ; R)$. This document may be retrieved via the EPAPS homepage (http://www.aip.org/pubservs/epaps.html) or from ftp.aip.org in the directory /epaps/. See the EPAPS homepage for more information

${ }^{48}$ W. M. Westgate, A. M. Simas, and V. H. Smith, Jr., J. Chem. Phys. 83, 4054 (1985).

${ }^{49}$ A. J. Thakkar, A. L. Wonfor, and W. A. Pedersen, J. Chem. Phys. 87, $1212(1987)$

${ }^{50}$ T. Koga, H. Matsuyama, H. Inomata, E. Romera, J. S. Dehesa, and A. J. Thakkar, J. Chem. Phys. 109, 1601 (1998).

${ }^{51}$ A. J. Thakkar, J. Chem. Phys. 86, 5060 (1987).

${ }^{52}$ A. J. Thakkar and W. A. Pedersen, Int. J. Quantum Chem., Symp. 23, 245 (1989).

${ }^{53}$ A. J. Thakkar, J. W. Liu, and W. J. Stevens, Phys. Rev. A 34, 4695 (1986).

${ }^{54}$ A. J. Thakkar, J. W. Liu, and G. C. Lie, Phys. Rev. A 36, 5111 (1987).

${ }^{55}$ A. J. Thakkar and H. Tatewaki, Phys. Rev. A 42, 1336 (1990).

${ }^{56}$ J. Wang and V. H. Smith, Jr., J. Phys. B 27, 5159 (1994).

${ }^{57}$ L. E. McMurchie and E. R. Davidson, J. Comput. Phys. 26, 218 (1978).

${ }^{58}$ M. Primorac, Int. J. Quantum Chem. 68, 305 (1998).

${ }^{59}$ J. O. Jensen, A. H. Carrieri, C. P. Vlahacos, D. Zeroka, H. F. Hameka, and C. N. Merrow, J. Comput. Chem. 14, 986 (1993).

${ }^{60}$ The Born-Oppenheimer energy from the 2400-term ECG wave function at $R=5.6$ bohr is -5.807483530 hartree which yields $-10.981 K$ of the interaction energy.

${ }^{61}$ A. N. Tripathi, R. P. Sagar, R. O. Esquivel, and V. H. Smith, Jr., Phys. Rev. A 45, 4385 (1992).

${ }^{62}$ H. Meyer, T. Müller, and A. Schweig, J. Mol. Struct.: THEOCHEM 360, 55 (1996).

${ }^{63}$ T. Koga and A. J. Thakkar, J. Phys. B 29, 2973 (1996).

${ }^{64}$ J. S. Lee, J. Chem. Phys. 66, 4906 (1977).

${ }^{65}$ K. T. Chung, X.-W. Zhu, and Z.-W. Wang, Phys. Rev. A 47, 1740 (1993).

${ }^{66}$ C. L. Pekeris, Phys. Rev. A 115, 1216 (1959).

${ }^{67}$ K. E. Banyard and J. C. Moore, J. Phys. B 10, 2781 (1977).

${ }^{68}$ P. Froelich and S. A. Alexander, Phys. Rev. A 42, 2550 (1990).

${ }^{69}$ F. J. Gálvez and I. Porras, Phys. Rev. A 51, 2857 (1995).

${ }^{70}$ I. R. Epstein, Phys. Rev. A 8, 160 (1973).

${ }^{71}$ A. Saenz, T. Asthalter, and W. Weyrich, Int. J. Quantum Chem. 65, 213 (1997).

${ }^{72}$ M. Abramovitz and I. A. Stegun, Handbook of Mathematical Functions (Dover, New York, 1974).

${ }^{73}$ G. P. M. Poppe and C. M. J. Wijers, ACM Trans. Math. Softw. 16, 47 (1990).

${ }^{74}$ G. W. F. Drake, Nucl. Instrum. Methods Phys. Res. B 31, 7 (1988).

${ }^{75}$ F. Arias de Saavedra, E. Buendía, and F. J. Gálvez, Z. Phys. D: At., Mol. Clusters 38, 25 (1996).

${ }^{76}$ J. Komasa, W. Cencek, and J. Rychlewski, Phys. Rev. A 52, 4500 (1995). 